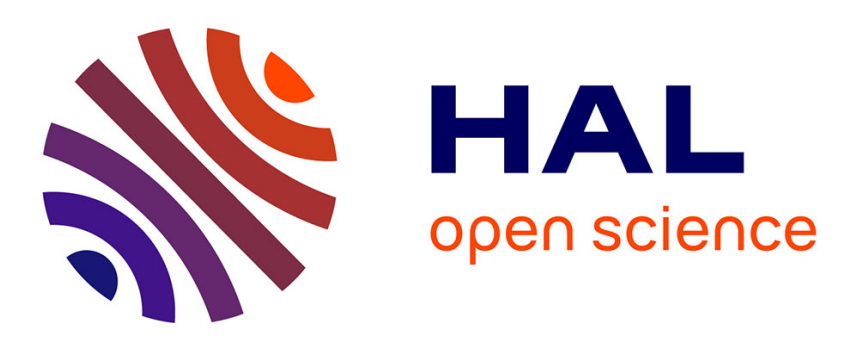

\title{
Modélisation du processus de compétition nutritive entre microorganismes: application aux Fusarium
}

Michèle Zegerman, Yvonne Couteaudier, Claude Alabouvette, Alain Pavé

\section{To cite this version:}

Michèle Zegerman, Yvonne Couteaudier, Claude Alabouvette, Alain Pavé. Modélisation du processus de compétition nutritive entre microorganismes: application aux Fusarium. Agronomie, 1987, 7 (10), pp.797-806. hal-00884955

\section{HAL Id: hal-00884955 https://hal.science/hal-00884955}

Submitted on 1 Jan 1987

HAL is a multi-disciplinary open access archive for the deposit and dissemination of scientific research documents, whether they are published or not. The documents may come from teaching and research institutions in France or abroad, or from public or private research centers.
L'archive ouverte pluridisciplinaire HAL, est destinée au dépôt et à la diffusion de documents scientifiques de niveau recherche, publiés ou non, émanant des établissements d'enseignement et de recherche français ou étrangers, des laboratoires publics ou privés. 


\section{Modélisation du processus de compétition nutri- tive entre microorganismes: application aux Fusarium}

Michèle ZEGERMAN, Yvonne COUTEAUdIER $\left({ }^{*}\right)$, Claude ALABOUVETTE $\left({ }^{*}\right)$ \& Alain PAVÉ

Laboratoire de Biométrie L. A. 243, Université Claude Bernard, Lyon I, 43, bd du 11-Novembre-1918, F 69622

Villeurbanne Cedex

(*) I.N.R.A., Station de Recherches sur la Flore pathogène dans le Sol, 17, rue Sully, F 21034 Dijon Cedex

Cet article présente les résultats obtenus après l'étude mathématique et biologique de la compétition nutritive entre Fusarium saprophytes et pathogènes. La dynamique de croissance de ces microorganismes suivant un modèle logistique, nous avons déterminé les paramètres de croissance de chaque souche par régression non linéaire. Une méthode efficace pour la détermination des paramètres de croissance en culture pure étant mise au point, nous pouvions alors nous intéresser au modèle de compétition. Construit sur l'hypothèse de partage d'un même substrat, il s'exprime simplement de la façon suivante :

$$
\begin{aligned}
& x+s \stackrel{\alpha_{1}}{\rightarrow}\left(1+R_{1}\right) x \\
& y+s \stackrel{\alpha_{2}}{\rightarrow}\left(1+R_{2}\right) y .
\end{aligned}
$$

Après une brève étude mathématique, le modèle est confronté aux données biologiques. Son adéquation avec ces dernières nous permet de confirmer l'hypothèse biologique d'une compétition nutritive. De plus une caractéristique importante réside dans la détermination d'un indice de compétition, spécifique du couple de souches étudié, dont le calcul peut se faire simplement à partir de la croissance de chacune des souches en culture pure ; l'issue de la compétition est ensuite déterminée par le rapport des concentrations d'inoculum initiales. Cette recherche suggère de nouvelles voies pour l'étude des interactions microbiennes utiles à la mise au point de procédés de lutte biologique.

Mots clés additionnels : Sol, dynamique des populations, lutte biologique.

Modeling of intrageneric competition for nutrients between Fusarium strains.

This paper presents the results obtained while studying intrageneric competition for nutrients between pathogenic and non-pathogenic Fusarium strains, from both mathematical and biological points of view. The population dynamics of these Fusarium strains in simple culture follow a logistic model and the parameters of growth may be determined by using a non-linear regression including a weighting term. Knowing the growth parameters of the strains in single culture and the initial concentration of each strain, it is possible to study the interaction between two strains growing together. The proposed model is based on the assumption that strains are competing for the same substrate. This model can be expressed in simple chemical terms as follow :

$$
\begin{aligned}
& x+s \stackrel{\alpha_{1}}{\rightarrow}\left(1+R_{1}\right) x \\
& y+s \stackrel{\alpha_{2}}{\rightarrow}\left(1+R_{2}\right) y .
\end{aligned}
$$

After a brief mathematical study, the model was fitted to experimental data. The satisfactory fit of the mathematical model to biological results confirmed the validity of the hypothesis of competition for a substrate. An important feature is that this model includes an index of competition, specific to the Fusarium strains studied. The determination of growth rate in single culture is sufficient to calculate this competition index. This research has led to new approaches for microbiological interaction studies useful for biological control. 


\section{INTRODUCTION}

Dans le domaine de l'utilisation des microorganismes pour la lutte biologique, il a été démontré que l'introduction dans un sol sensible désinfecté de souches de Fusarium non pathogènes permet de réduire considérablement la gravité d'une fusariose vasculaire (COUTEAUDIER et al., 1985). Ces résultats obtenus avec un mélange de Fusarium non pathogènes conduisent à rechercher les critères permettant de sélectionner les souches les plus efficaces. L'analyse des cinétiques de croissance de différentes populations de Fusarium dans le sol permet d'aborder l'étude de deux de ces critères : l'aptitude à coloniser le sol et l'aptitude à entrer en compétition avec l'agent pathogène. Par ailleurs, l'analyse des dynamiques de populations dans le sol donne accès à l'étude du déterminisme de la compétition; en effet, l'hypothèse d'une interaction de type compétition nutritive s'exerçant entre populations non pathogènes de Fusarium et populations pathogènes de Fusarium oxysporum (Schelcht) Sn. et H. a été proposée lors des travaux consacrés aux mécanismes de résistance des sols de Châteaurenard aux fusarioses vasculaires (Alabouvette, 1986).

Il est possible de suivre expérimentalement les dynamiques de différentes populations de Fusarium introduites dans le sol à des niveaux de concentration déterminés. Des résultats préliminaires ont montré que le modèle logistique peut s'ajuster aux cinétiques de croissance de différentes populations de $F$. oxysporum et $F$. solani (Mart.) (Appel et Wr.) Sn. et H. Néanmoins, la mise en œuvre de ce modèle a conduit à une assez forte imprécision des valeurs estimées des densités d'inoculum initiales et du taux de croissance (SON, 1985 ; Couteaudier et al., 1986). Pour pallier cet inconvénient, nous proposons en premier lieu dans cet article une méthode d'estimation précise des paramètres de croissance. La mise au point de cette procédure était nécessaire à l'élaboration d'un modèle mathématique de compétition entre 2 populations. Le modèle choisi a été formulé de façon simple par PAVF́ \& RECHENMANN (1986) qui exploitent la parenté existant entre les modèles de la cinétique chimique et ceux de la dynamique des populations. Nous avons donc confronté ce modèle mathématique aux données biologiques obtenues dans une expérimentation basée sur l'inoculation d'un sol par 2 populations de Fusarium dont on fait varier le niveau d'inoculum initial et le rapport des concentrations.

\section{MATÉRIEL ET MÉTHODE}

\section{A. Matériel biologique et analyse des populations}

Des échantillons de $300 \mathrm{~g}$ de sol tamisé à $2 \mathrm{~mm}$ sont traités à la chaleur 3 fois à $24 \mathrm{~h}$ d'intervalle par autoclavage d'une demi-heure à $120^{\circ} \mathrm{C}$. Les populations de Fusarium sont ensuite apportées au sol sous forme de propagules conservées dans du talc (TELLO-MARQUINA \& AlABOUVETTE, 1984); le talc support physicochimique inerte, permet d'effectuer des dilutions précises de l'inoculum utilisé. Une bonne répartition de l'inoculum dans le sol est assurée par agitation mécani- que pendant $15 \mathrm{mn}$ (mélangeur Turbula). L'humidité étant ajustée à un potentiel hydrique de -1 bar, les échantillons sont conservés dans une pièce thermostatée à $23{ }^{\circ} \mathrm{C}$. L'analyse des populations fusariennes est effectuée à intervalles de temps réguliers ; des suspensions dilutions successives au dixième sont réalisées sur des échantillons de $10 \mathrm{~g}$ de terre et réparties en boîtes de Petri par fractions de $1 \mathrm{ml}$ à raison de 10 boîtes par dilution. Les milieux utilisés sont le milieu à base d'extrait de malt et un milieu malt acidifié additionné de bénomyl à la concentration de $5 \mathrm{mg} / \mathrm{l}$.

Expérimentation 1: Etude de la cinétique de croissance de la souche Foln 3 (Fusarium oxysporum $f . s p$. lini, agent de la fusariose vasculaire du lin) en sol de Châteaurenard. Cinq échantillons de sol traité à la chaleur ont été inoculés à une concentration d'inoculum initiale de $1.10^{3}$ propagules $/ \mathrm{g}$ de sol sec.

Expérimentation 2: Etude de l'interaction entre 2 populations. Les souches utilisées sont :

- Foln 3.5: F. oxysporum lini résistant au bénomyl,

- Fs 2 : F. solani isolé du sol résistant de Châteaurenard.

Chaque souche est inoculée au sol de Dijon traité à la chaleur soit en culture pure, soit en culture mixte dans différentes proportions :

$\begin{array}{cc}\mathrm{x}_{0}(\text { Fs } 2) & \mathrm{y}_{0} \text { (Foln 3) } \\ (\text { microorganismes par g de sol) } \\ 0,610^{3} & - \\ 7,210^{3} & - \\ - & 0,610^{3} \\ - & 8,210^{3} \\ 6,210^{3} & 0,710^{3} \\ 5,710^{3} & 7,610^{3} \\ 0,610^{3} & 8,010^{3}\end{array}$

Les caractéristiques physico-chimiques des sols utilisés dans les 2 expériences ont été publiées précédemment (CORMAN et al., 1986).

\section{B. Estimation des paramètres}

La croissance en fonction du temps $t$ et de la biomasse $x$ peut s'exprimer sous la forme logistique :

$$
\begin{aligned}
& \frac{\mathrm{dx}}{\mathrm{dt}}=\mu \mathrm{x}\left(1-\frac{1}{\mathrm{~K}} \mathrm{x}\right) \quad \mu: \text { taux de croissance } \\
& \mathrm{K} \text { : capacité limite du milieu } \\
& \mathrm{x} \text { : densité de population. }
\end{aligned}
$$

Pour chaque souche un ajustement non linéaire (PAVÉ, 1980) basé sur le critère des moindres carrés permet d'estimer les paramètres suivants :

- le taux de croissance,

- le plateau (ou capacité limite du milieu),

- la valeur de l'inoculum initial.

Le programme d'ajustement écrit en FORTRAN 77 comprend les procédures suivantes (CORMAN \& PAVÉ, 1983) :

- estimations initiales par interpolation parabolique sur 3 points successifs ;

- estimation des paramètres par régression non linéaire. Adaptation de la procédure de Gauss - Mar- 
quardt proposée par Fletcher qui est utilisée pour minimiser la somme des carrés suivante :

$\mathrm{S}(\vec{\theta})=\sum_{i=1}^{n}\left(\mathrm{y}_{\mathrm{i}}-\mathrm{f}\left(\mathrm{x}_{\mathrm{i}}, \vec{\theta}\right)\right)^{2}$

$x_{i}, y_{i} \quad:$ i-ième point expérimental

$\mathrm{f}\left(\mathrm{x}_{\mathrm{i}}, \vec{\theta}\right)$ : fonction de prédiction associée à $\mathrm{x}_{\mathrm{i}}$ et à $\vec{\theta}$ (vecteur des paramètres).

Le traitement informatique a été réalisé sur matériel Data General S 140.

La mise en ouvre du modèle logistique donne des valeurs estimées des densités d'inoculum initial très supérieures aux valeurs observées ainsi qu'une trop forte imprécision de l'ajustement en début de croissance et par conséquent dans l'estimation du taux de croissance.

Afin de pallier cet inconvénient une autre procédure de régression non linéaire a été envisagée telle que le poids accordé à chaque observation soit dépendant de la précision de cette observation. Le programme d'ajustement utilise alors la somme des carrés suivante :

$S(\vec{\theta})=\sum_{i=1}^{n} w_{i}\left(y_{i}-f\left(x_{i}, \vec{\theta}\right)\right)^{2}$

$w_{\mathrm{i}}$ : terme de pondération.

\section{Le modèle de compétition}

L'hypothèse d'une interaction de type compétition a conduit à l'utilisation des modèles classiques de LOTKA (1932) et VOLTERRA (1931). Le modèle proposé (PAVÉ \& RECHENMANN, 1986) pour l'étude de l'interaction est le suivant :

$$
\begin{aligned}
& x+s \stackrel{\mathrm{x}_{1}}{\rightarrow}\left(1+\mathrm{R}_{1}\right) \mathrm{x} \\
& \mathrm{y}+\mathrm{s} \stackrel{\stackrel{\mathrm{x}_{2}}{\rightarrow}}{\rightarrow}\left(1+\mathrm{R}_{2}\right) \mathrm{y}
\end{aligned}
$$

avec $\alpha_{1}$ et $\alpha_{2}$ interprétés comme les vitesses de croissance des populations $x$ et $y, R_{1}$ et $R_{2}$ rendements de croissance des populations sur le substrat s. Ce système décrit donc la croisssance des 2 populations microbiennes $\mathrm{x}$ et $\mathrm{y}$ sur un même substrat $\mathrm{s}$ avec des rendements respectifs de $R_{1}$ pour $x$ et $R_{2}$ pour $y$.

\section{Hypothèses liées au modèle}

Les 2 populations sont supposées suivre une croissance logistique :

- sur un milieu ne contenant que l'espèce $\mathrm{x}$ on peut écrire :

$$
\left(s_{0}-s\right)=1 / R_{1}\left(x-x_{0}\right)
$$

- sur un milieu ne contenant que l'espèce y on peut écrire :

$$
\left(s_{0}-s\right)=1 / R_{2}\left(y-y_{0}\right) \text {. }
$$

Les 2 populations partagent le même substrat lorsqu'elles sont en présence :

$s=s_{0}-1 / R_{1}\left(x-x_{0}\right)-1 / R_{2}\left(y-y_{0}\right)$
La mortalité de chacune des 2 espèces reste négligeable.

L'expression du modèle peut donc se donner sous le formalisme différentiel suivant :

$$
\begin{aligned}
& \frac{\mathrm{dx}}{\mathrm{dt}}=\alpha_{1} \mathrm{R}_{1} \mathrm{xs} \\
& \frac{\mathrm{dy}}{\mathrm{dt}}=\alpha_{2} \mathrm{R}_{2} \mathrm{ys} \\
& \frac{\mathrm{ds}}{\mathrm{dt}}=-\alpha_{1} \mathrm{xs}-\alpha_{2} \mathrm{ys} .
\end{aligned}
$$

Soit en remplaçant s par sa valeur (1) :

$$
\begin{aligned}
& \frac{d x}{d t}=\alpha_{1}\left(K-x-\frac{R_{1}}{R_{2}} y\right) x \\
& \frac{d y}{d t}=\alpha_{2} \frac{R_{2}}{R_{1}}\left(K-x-\frac{R_{1}}{R_{2}} y\right) y
\end{aligned}
$$

avec

$$
\mathrm{K}=\mathrm{R}_{1} \mathrm{~s}_{0}+\mathrm{x}_{0}+\mathrm{R}_{1} / \mathrm{R}_{2} \mathrm{y}_{0}
$$

Le point d'équilibre est donné par $\mathrm{dx} / \mathrm{dt}=\mathrm{dy} / \mathrm{dt}=0$. On obtient :

$$
\begin{aligned}
& \alpha_{1}\left(K-x-R_{1} / R_{2} y\right) x=0 \\
& \alpha_{2} R_{2} / R_{1}\left(K-x-R_{1} / R_{2} y\right) y=0
\end{aligned}
$$

Les solutions sont :

$-\mathrm{x}=\mathrm{y}=0$;

$-\mathrm{x} \neq \mathrm{y} \neq 0$, on a alors la droite d'équilibre dépendante des conditions initiales : $K-x-R_{1} / R_{2} y=0$. Il n'y a donc pas de point d'équilibre unique dans l'espace des variables $(x, y)$, mais un ensemble de points d'équilibre sur la droite d'équilibre précédemment énoncée ;

$-\mathrm{x}=0$ et $\mathrm{y} \neq 0$, on obtient $\mathrm{y}=\mathrm{K} \mathrm{R} / \mathrm{R}_{2}$;

$-x \neq 0$ et $y=0$, on obtient $x=K$.

On peut visualiser ceci en représentant la droite d'équilibre et l'évolution du couple dans le plan de phase (fig. 1).

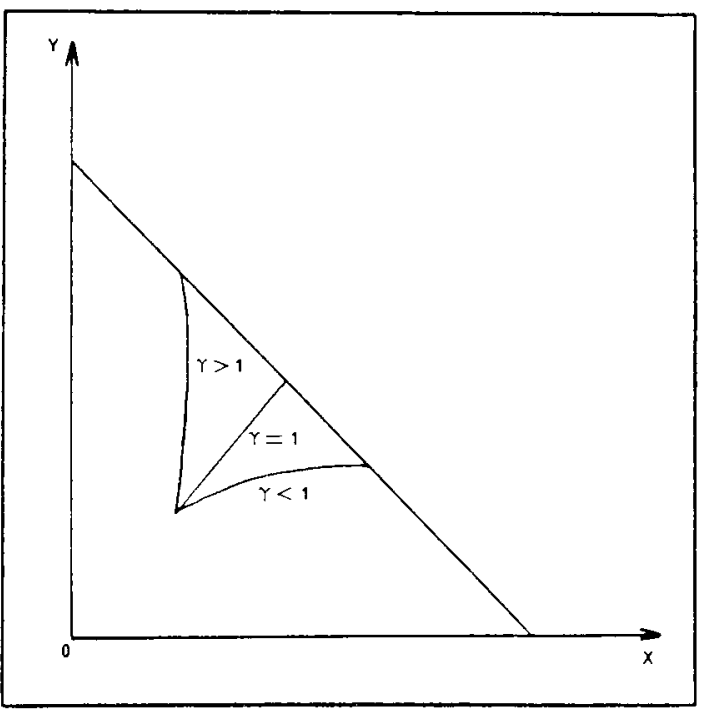

Figure 1

Plan de phase théorique.

Theoretical phase plane. 
L'équation de la trajectoire du couple $\mathrm{x}$, y dans le plan de phase est alors donnée par :

$$
\mathrm{y}=\mathrm{y}_{0}\left(\frac{\mathrm{x}}{\mathrm{x}_{0}}\right)^{y}
$$

avec

$$
\gamma=\gamma \frac{\alpha_{2} R_{2}}{\alpha_{1} R_{1}} .
$$

L'intersection de cette courbe avec la droite d'équilibre nous donne le point d'équilibre auquel chaque couple $\mathrm{x}, \mathrm{y}$ doit aboutir. L'issue de la compétition dépend de la valeur de l'exposant $\gamma$.

En culture pure l'expression des plateaux des souches est donnée par $K_{1}=R_{1} s_{0}+x_{0}$ et $K_{2}=R_{2} s_{0}+y_{0}$, points d'équilibre du modèle logistique d'où les expressions de $R_{1}$ et $R_{2}$ :

$$
\mathrm{R}_{1}=\frac{\mathrm{K}_{1}-\mathrm{x}_{0}}{\mathrm{~s}_{0}} \text { et } \mathrm{R}_{2}=\frac{\mathrm{K}_{2}-\mathrm{y}_{0}}{\mathrm{~s}_{0}}
$$

De par l'hypothèse d'un partage de même substrat il vient :

$$
\frac{\mathrm{R}_{1}}{\mathrm{R}_{2}} \approx \frac{\mathrm{K}_{1}}{\mathrm{~K}_{2}} \text { d'où } \gamma=\frac{\alpha_{2} \mathrm{R}_{2}}{\alpha_{1} \mathrm{R}_{1}}=\frac{\mu_{2}}{\mu_{1}} .
$$

L'exposant $\gamma$ est donc le rapport de paramètres que l'on sait estimer en culture pure à savoir les taux de croissance. On suppose de plus que les termes $\mathrm{x}_{0}$ et $\mathrm{y}_{0}$ sont négligeables devant $K_{1}$ et $K_{2}$, et, de par l'homogénéité des échantillons, que la grandeur $\mathrm{s}_{0}$ est constante d'une expérience à l'autre.

\section{RÉSULTATS}

\section{A. Expérimentation 1}

Les résultats obtenus après ajustement de la croissance de Foln 3 au modèle logistique, avec ou sans pondération, sont mentionnés dans le tableau 1. La décroissance finale observée sur la figure 2 n'est pas significative et ne remet pas en cause l'utilisation du modèle logistique validé dans des expérimentations préalables (COUTEAUDIER et al., 1986).

La pondération par la variance se traduit par une grande variabilité dans l'estimation des paramètres; elle ne sera donc pas retenue. La représentation graphique de la dynamique de croissance de Foln 3, pour les 5 répétitions et pour les 3 types d'ajustement (fig. 2) montre une perte de précision sur le plateau, principalement après pondération par les écarts-types. En contrepartie, la pondération par les écarts-types apporte un gain de précision dans l'estimation du taux de croissance; la valeur de l'inoculum initial qui ne subit aucun traitement devient alors très proche de sa valeur nominale : le gain est supérieur à celui apporté par pondération par les données. Ce dernier type d'ajustement est un compromis entre la procédure sans pondération et celle comprenant une pondération par les écarts-types.

Le tracé des ellipses d'inertie (fig. 3) pour le plateau et le taux de croissance nous confirme ces résultats.

On peut aussi donner une évaluation de la reproductibilité des estimations à partir de l'écart-type de la moyenne rapporté à cette dernière. Les résultats exprimés en pourcentage sont résumés tableau 2.

On observe que l'amélioration de la précision sur le taux de croissance est nette après l'ajustement prenant

\begin{tabular}{|c|c|c|c|c|}
\hline Répétition & Type de pondération & Plateaux $\left(x 10^{7}\right)$ & Taux de croissance & Inoculum calculé \\
\hline 1 & aucune & 0,180 & 0,533 & $0,23510^{5}$ \\
\hline 1 & données & 0,158 & 0,914 & $1,573 \quad 10^{3}$ \\
\hline 1 & écarts-types & 0,156 & 0,986 & $1,077 \quad 10^{3}$ \\
\hline 1 & variance & 0,221 & 0,533 & $0,136 \quad 10^{\circ}$ \\
\hline 2 & aucune & 0,191 & 0,960 & $1,703 \quad 10^{3}$ \\
\hline 2 & données & 0,186 & 1,023 & $0,99510^{3}$ \\
\hline 2 & écarts-types & 0,191 & 1,026 & $1,04410^{3}$ \\
\hline 2 & variance & 0,171 & 0,915 & $0,606 \quad 10^{4}$ \\
\hline 3 & aucune & 0,181 & 1,068 & $0,513 \quad 10^{3}$ \\
\hline 3 & données & 0,179 & 1,016 & $0,726 \quad 10^{3}$ \\
\hline 3 & écarts-types & 0,181 & 0,995 & $1,035 i \cap^{3}$ \\
\hline 3 & variance & 0,165 & 0,796 & $0,113 \quad 10^{\circ}$ \\
\hline 4 & aucune & 0,174 & 2,539 & $0,411 \quad 10=$ \\
\hline 4 & données & 0,173 & 2,899 & $0,245 \quad 10^{-3}$ \\
\hline 4 & écarts-types & 0,186 & 0,980 & $0,97910^{3}$ \\
\hline 4 & variance & 0,254 & 0,487 & $0,999 \quad 10^{3}$ \\
\hline 5 & aucune & 0,193 & 1,743 & 1,838 \\
\hline 5 & données & 0,190 & 1,441 & 1,887 \\
\hline 5 & écarts-types & 0,207 & 0,990 & $0,911 \quad 10^{3}$ \\
\hline 5 & variance & 0,048 & 1,195 & $0,59610^{4}$ \\
\hline
\end{tabular}

TABLEAU 1

Valeurs des paramètres de croissance de la souche Foln 3 en culture pure après ajustement du modèle logistique avec ou sans pondération. Growin parameter values of Foln 3 after non-linear fitting of a logistic model. 

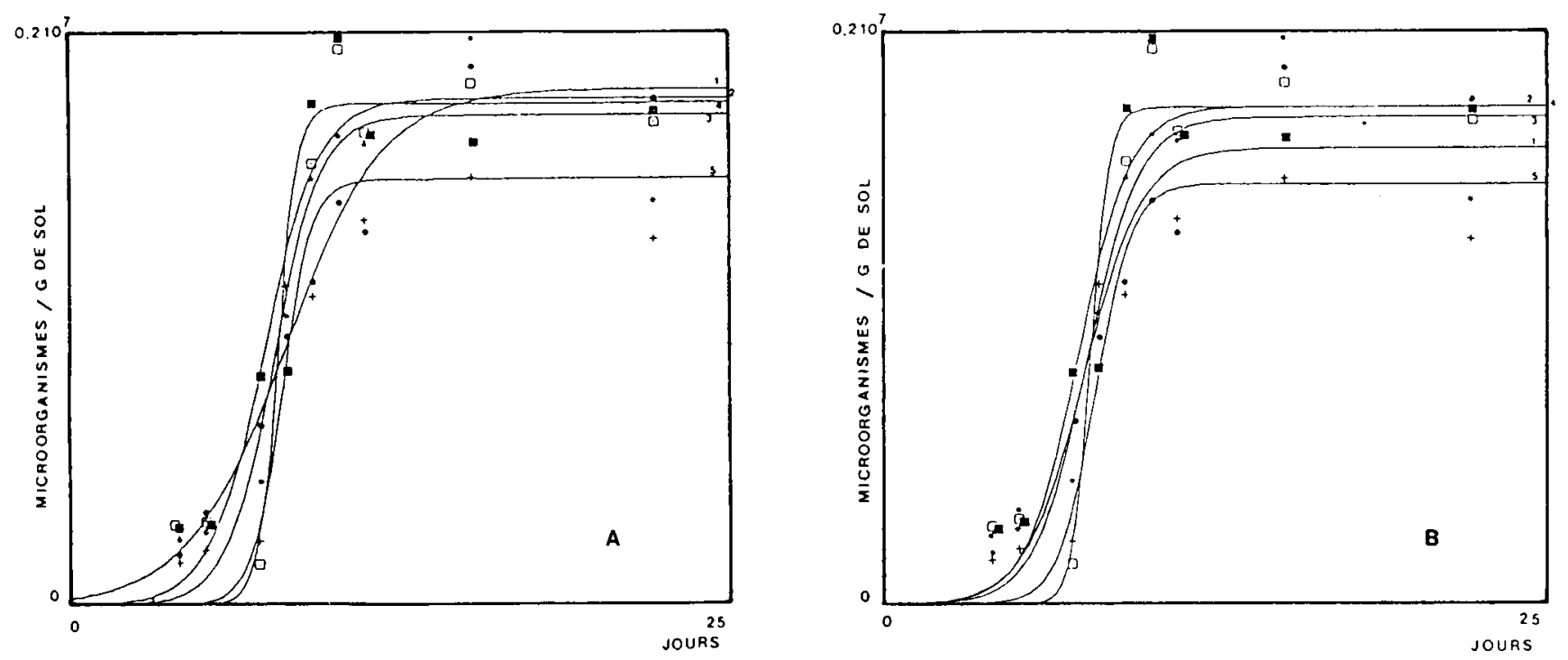

\section{Figure 2}

Tracé des ajustements de la croissance de Foln 3 au modèle logisti$q u e\left(\bullet\right.$ répétition $l, \square$ répétition $2,{ }^{*}$ répétition $3, \square$ répétition 4 , + répétition 5)

A sans pondération

$B$ après pondération par les données

$C$ après pondération par les écarts-types.

Growth kinetics of Foln 3 after fitting to logistic model with nonlinear procedure $\left(\bullet\right.$ replicate $1, \square$ replicate $2,{ }^{*}$ replicate $3, \square$ replicate $4,+$ replicate 5$)$

$A$ without weighting term

$B$ weighted by data

$C$ weighted by standard deviation.

en compte la pondération par les écarts-types, même si l'on perd un peu de précision sur l'estimation du plateau. Malgré son caractère quelque peu empirique nous porterons donc notre choix sur cette méthode d'estimation car c'est le paramètre taux de croissance que nous privilégions.

\section{B. Expérimentation 2}

Pour l'évolution en culture mixte des densités de population de Foln 3.5 et Fs 2 apportées dans le milieu à des concentrations différentes (fig. 4), on notera que la souche introduite à la plus forte dose d'inoculum atteint le plateau le plus élevé.

Pour les paramètres de croissance des souches en culture pure (tabl. 3), on remarque que pour des taux de croissance voisins, les plateaux atteints par chacune des 2 souches sont différents.

Pour les diverses valeurs des coefficients d'interaction (tabl. 4), on distingue :

- $\gamma_{\text {hhéor }}$ déterminé à partir des données expérimen-

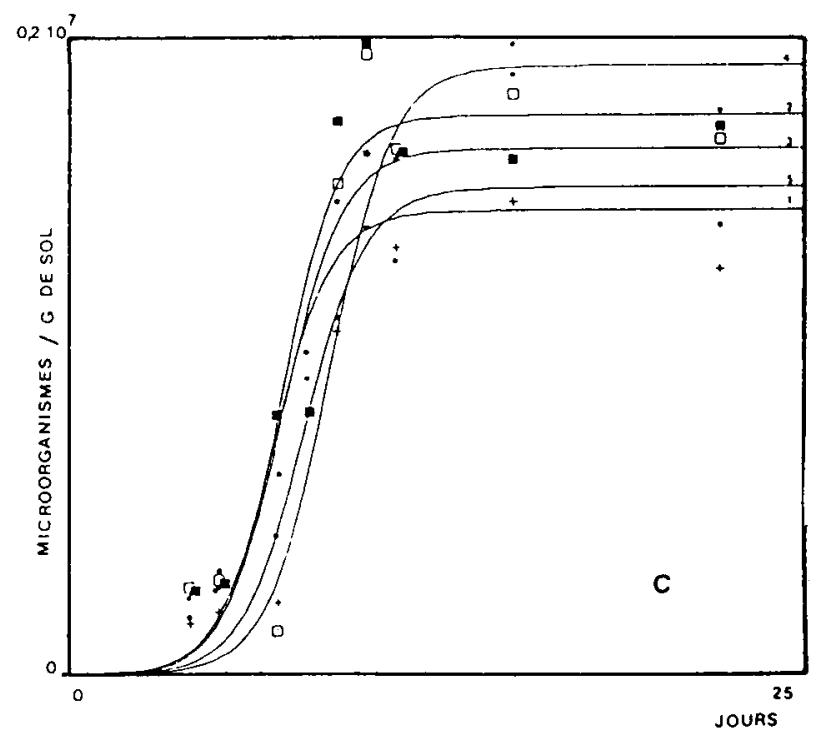

tales issues de la croissance des microorganismes en culture pure,

- $\gamma_{\text {exp }}$ déterminé à partir des données expérimentales issues de la croissance des microorganismes en culture mixte. La détermination est réalisée par régression logarithmique de y sur $x$ en reprenant l'équation (2), les coefficients de corrélation sont mentionnés sur le même tableau. Leurs valeurs très satisfaisantes vont dans le sens de la validation du modèle. On observe que quel que soit le rapport des concentrations initiales, $\gamma_{\exp }$ n'est pas différent de $\gamma_{\text {théor }}$.

\section{Point d'équilibre}

Connaissant la valeur du paramètre $\gamma_{\text {théor }}$, on peut tracer la trajectoire du couple dans le plan de phase (fig. 5). La trajectoire expérimentale figure également sur ces graphiques.

Le point d'équilibre étant donné par l'intersection de la trajectoire du couple et de la droite d'équilibre il est aisé de le déterminer par la méthode de Newton (BARANGER, 1977). 

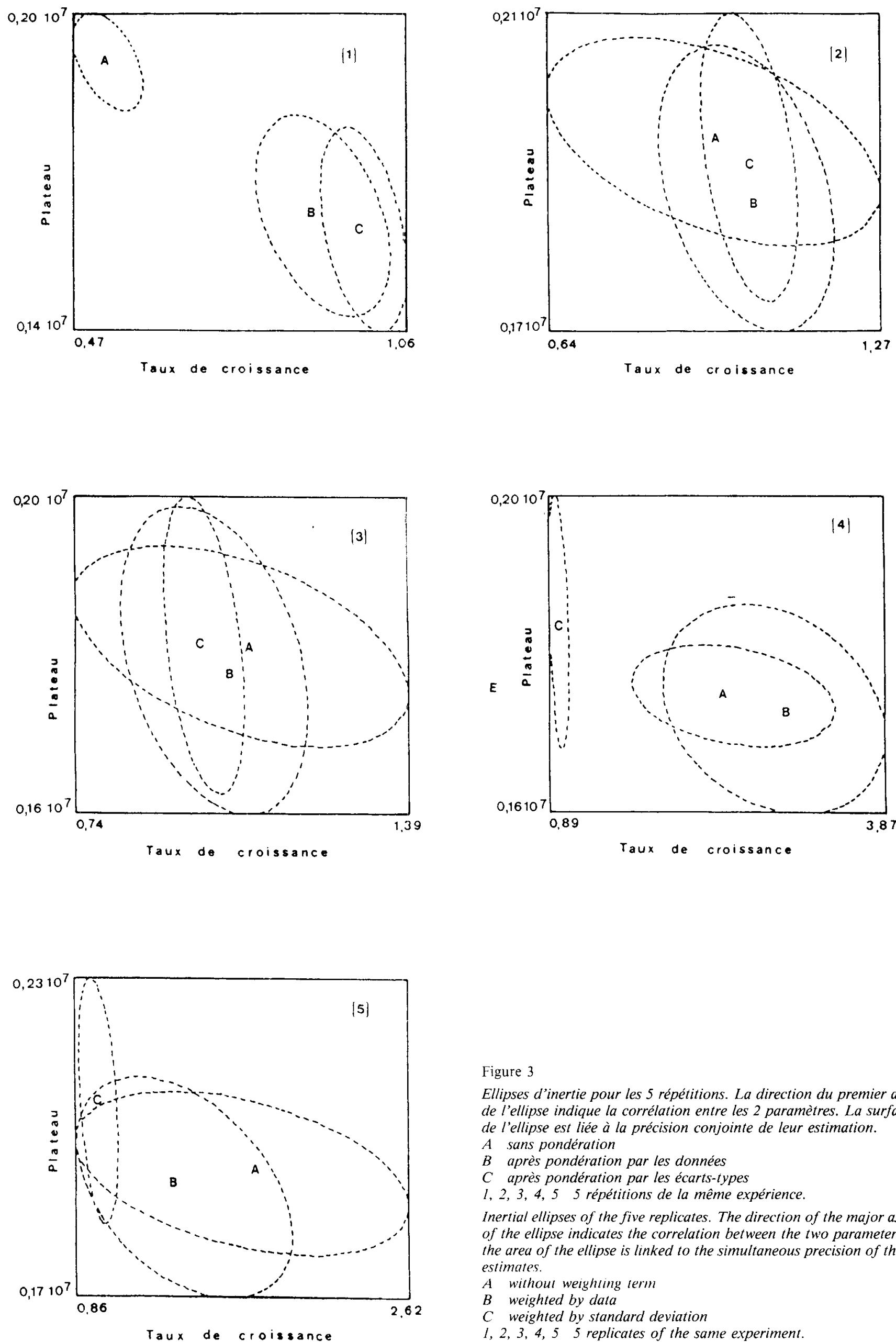

Figure 3

Ellipses d'inertie pour les 5 répétitions. La direction du premier axe de l'ellipse indique la corrélation entre les 2 paramètres. La surface de l'ellipse est liée à la précision conjointe de leur estimation.

$A$ sans pondération

$B$ après pondération par les données

$C$ après pondération par les écarts-types

$I, 2,3,4,55$ répétitions de la même expérience.

Inertial ellipses of the five replicates. The direction of the major axis of the ellipse indicates the correlation between the two parameters; the area of the ellipse is linked to the simultaneous precision of their estimates.

A without weighing term

$B$ weighted by data

$C$ weighted by standard deviation

$1,2,3,4,55$ replicates of the same experiment. 

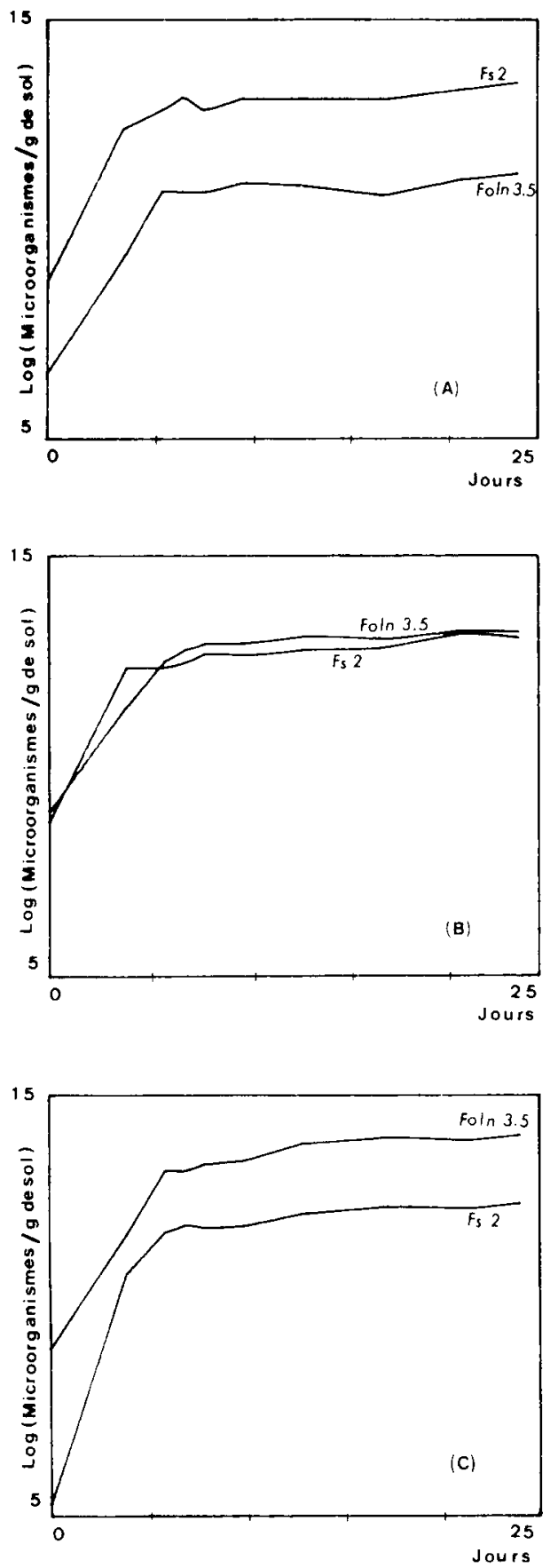

Figure 4

Evolution des densités de population du couple FS 2/Foln 3.5 exprimée dans une échelle logarithmique en fonction du temps, pour 3 rapports de concentration.

$\begin{array}{lll} & \text { FS } 2 & \text { Foln } 3.5 \\ A & 6,210^{3} & 0,710^{3} \\ B & 5,710^{3} & 7,610^{3} \\ C & 0,610^{3} & 8,010^{3}\end{array}$

Growth kinetics for the two strains Fs 2 and Foln 3.5, expressed on a logarithmic scale against time.

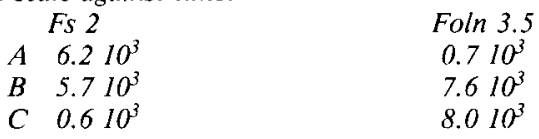

Les coordonnées en $\mathrm{x}$ et $\mathrm{y}$ du point d'équilibre sont (en nombre de microorganismes/g de sol) :

$\begin{array}{ccc}\text { essai } & x & y \\ 1 & 453000 & 49400 \\ 2 & 341000 & 376200 \\ 3 & 221000 & 932000\end{array}$

\section{TABLEAU 2}

Valeurs en pourcentage des coefficients de variation traduisant la reproductibilité des divers modes d'ajustement.

Variation coefficients $(\%)$ expressing the reproductibility of different fitting procedures.

\begin{tabular}{lcc}
\hline \multicolumn{1}{c}{ Ajustements } & Plateaux & Taux de croissance \\
\hline Sans pondération & 4,25 & 57,40 \\
Pondéré par les données & 7,01 & 56,90 \\
Pondéré par les écarts-types & 10,04 & 1,82 \\
Pondéré par la variance & 41,26 & 29,50 \\
\hline
\end{tabular}

\section{TABLEAU 3}

Valeurs des paramètres de croissance en culture pure des souches Fs 2 et Foln 3.5.

Growth parameters of Foln 3.5 and Fs 2 in pure culture.

\begin{tabular}{ccccc}
\hline Souches & $\begin{array}{c}\text { Inoculum } \\
\text { nominal }\end{array}$ & Plateaux & $\begin{array}{c}\text { Taux de } \\
\text { croissance }\end{array}$ \\
\hline Fs 2 & $0,610^{3}$ & $0,76210^{6} \pm 0,925$ & $10^{5}$ & $0,922 \pm 0,066$ \\
Fs 2 & $6,210^{3}$ & $0,47210^{6} \pm 0.21810^{5}$ & $0,954 \pm 0,131$ \\
Foln 3 & $0,710^{3}$ & $0,12210^{7} \pm 0,55510^{5}$ & $0,981 \pm 0,103$ \\
Foln 3 & $8,010^{3}$ & $0,13410^{7} \pm 0,73510^{5}$ & $0,835 \pm 0,035$ \\
\hline
\end{tabular}

\section{TABLEAU 4}

Valeurs des coefficients d'interaction "̈ et "̈hor déterminés à partir de la confrontation des souches Foln 3.5 et Fs 2 en culture mixte. Interactive coefficient values $\gamma_{\text {exp }}$ and $i$ theor determined from mixed culture.

\begin{tabular}{cccc}
\hline Essais & exp & iheor & $\begin{array}{c}\text { Coefficient } \\
\text { de corrélation }\end{array}$ \\
\hline 1 & $1,014 \pm 0,079$ & $1,028 \pm 0,177$ & 0,976 \\
2 & $0,976 \pm 0,099$ & $0,875 \pm 0,125$ & 0,961 \\
3 & $0,851 \pm 0,054$ & $0,906 \pm 0,075$ & 0.971 \\
\hline
\end{tabular}

Le point d'équilibre ne semble jamais atteint par le couple $\mathrm{x}, \mathrm{y}$. Ceci peut s'expliquer par l'accumulation des imprécisions sur les grandeurs considérées : le taux de croissance, les plateaux ainsi que les mesures expérimentales. Compte tenu de ces critères la tendance d'évolution reste conservée.

\section{DISCUSSION}

\section{A. Expérimentation 1}

La comparaison des 4 types d'ajustement nous enseigne que la pondération par les écarts-types donne des meilleurs résultats quant à l'estimation du paramètre dont nous voulons privilégier l'emploi. Ceci peut s'expliquer par une grande variabilité de la variance et par un ordre de grandeur des écarts-types proche de celui des données.

Il convient de noter que nos observations ne sont pas en accord avec la littérature. Dans cette dernière, on 

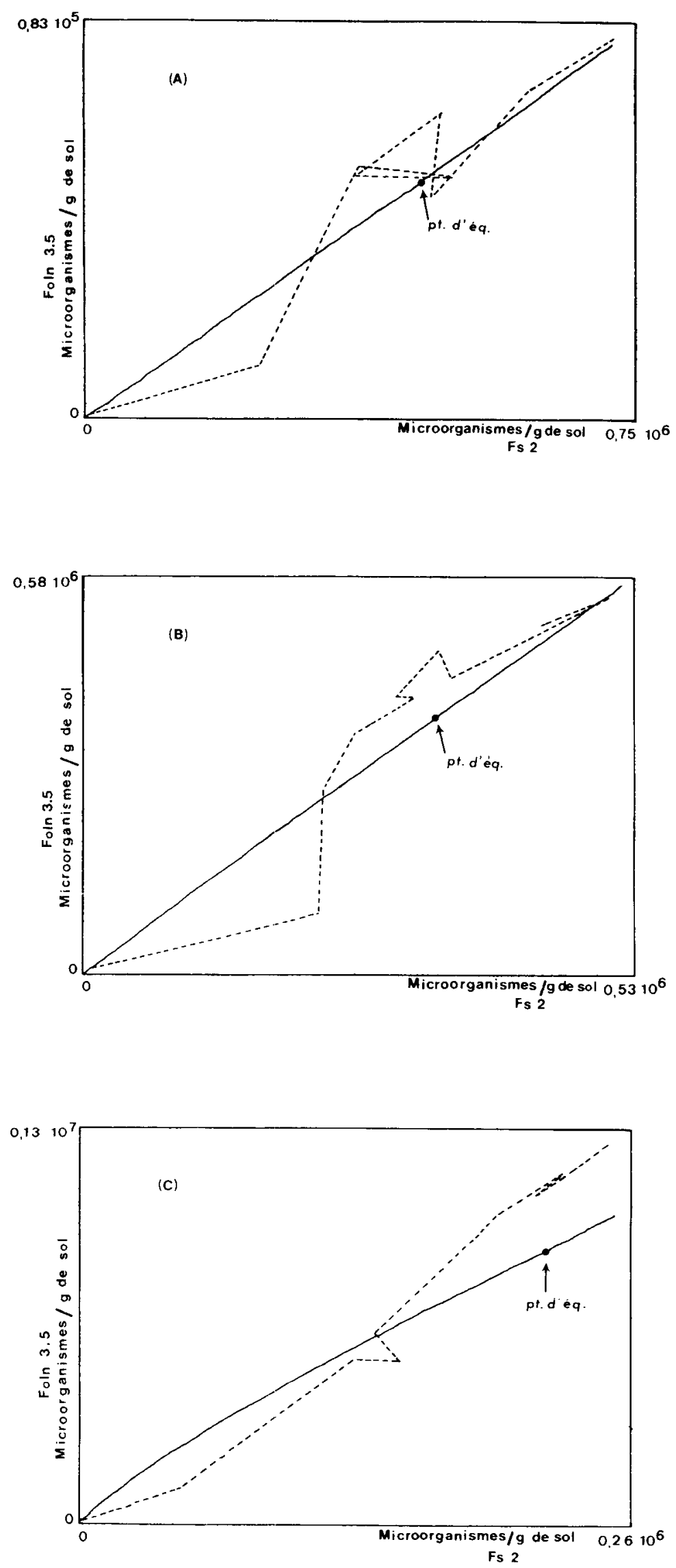

Figure 5

Evolution des densités (--- observée - théorique) de population de Fs 2/Foln 3.5 dans le plan de phase pour les 3 rapports de concentration.

$\begin{array}{llc} & F s 2 & \text { Foln } 3.5 \\ A & 6,210^{3} & 0,710^{3} \\ B & 5,710^{3} & 7,610^{3} \\ C & 0,610^{3} & 8,010^{3}\end{array}$

Growth kinetics of Fs 2/Foln 3.5 in the phase plante (--- observed theoretical).

$\begin{array}{llr} & F s 2 & \text { Foln } 3.5 \\ A & 6.210^{3} & 0.710^{3} \\ B & 5.710^{3} & 7.610^{3} \\ C & 0.610^{3} & 8.010^{3}\end{array}$


trouve de nombreux exemples de pondération des données par la variance (FINNEY, 1978). Dans notre cas, ceci nous conduit à accorder un poids trop faible aux observations proches du plateau en raison de leur ordre de grandeur $10^{6}$ qui, élevé au carré, rend négligeable l'effet de ces mesures. La pondération par l'écart-type, du même ordre de grandeur que les données, est donc préférable.

\section{B. Expérimentation 2}

La valeur de l'exposant $\gamma$ est identique quel que soit son mode d'estimation. On note qu'elle est indépendante des conditions initiales ce qui vérifie une de nos hypothèses de départ.

Le coefficient $\gamma$ représente donc un indice de compétition caractéristique du couple de souches mises en confrontation. La connaissance des taux de croissance en culture pure étant la seule donnée nécessaire à sa détermination, il sera aisé de prévoir l'issue de différentes combinaisons mettant en jeu diverses souches dont on connaîtra le niveau des concentrations initiales. On pourra ainsi entreprendre la comparaison des souches pour leur aptitude à entrer en compétition avec un agent pathogène connu, établir un classement en fonction de cette aptitude et prévoir l'issue de la compétition entre 2 populations.

Dans notre étude sur le couple Foln 3.5/Fs 2, il $s$ 'avère que la valeur de cet exposant est proche de 1 (fig. 6). La dernière valeur de $\gamma=0,851$ peut s'expliquer par la sous-estimation de la population Fs 2 lorsqu'elle est inoculée à la concentration la plus faible. En effet la technique de dénombrement par suspensiondilution peut conduire à une estimation par défaut dans le dénombrement de la population la moins abondante. $\gamma$ proche de 1 signifie qu'il y a proportionnalité entre la valeur du rapport $x / y$ à l'instant $t$ et la valeur du rapport $\mathrm{x}_{0} / \mathrm{y}_{0}$, rapport des concentrations d'inoculum initiales. Cette comparaison entre les rapports des états initiaux et ceux des états à l'équilibre sert également de base au modèle de compétition pour la nodulation entre souches de Rhizobium décrit par AMARGER \& LOBREAU (1982). Des valeurs de $\gamma$ différentes de la valeur 1 peuvent sans doute être obtenues en confrontant des souches différentes ou en introduisant une variation des facteurs environnementaux sur le couple Foln 3.5/Fs 2, par exemple la température ou l'humidité.

L'imprécision sur la détermination expérimentale du plateau ne nous permet pas de déterminer avec exactitude la valeur du point d'équilibre expérimental ; de plus le calcul du point d'équilibre théorique dépend des estimations des taux de croissance et des plateaux et est donc entaché d'erreur. En conséquence on ne peut pas observer une adéquation parfaite entre ces 2 valeurs mais simplement une tendance évolutive.

Toutefois les valeurs théoriques de $\gamma$ et les rapports des taux de croissance estimés en culture pure étant très proches nous pouvons estimer qu'il existe une adéquation entre le modèle mathématique et les données expérimentales.

Des expérimentations mettant en compétition des populations dont les taux de croissance en culture pure sont très différents devraient permettre de valider ou non le modèle proposé. Dans l'affirmative, l'hypothèse d'une compétition pour des éléments nutritifs sera confirmée et il sera alors possible d'en entreprendre la recherche. Ceci nécessite de se placer dans des conditions contrôlées et non plus en sol traité à la chaleur. L'utilisation d'un substrat inerte tel que la perlite additionnée d'un milieu synthétique est envisagé.

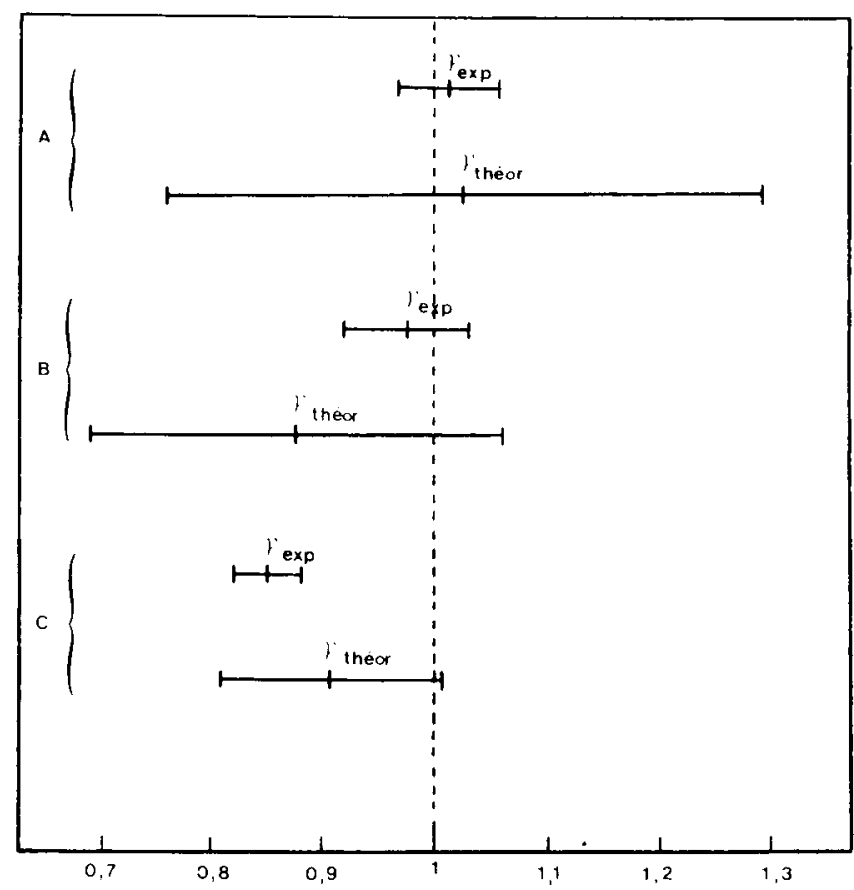

Figure 6

Valeurs des coefficients d'interaction expérimentaux et théoriques et leurs intervalles de confiance à 5. p. 100, pour les 3 rapports de concentration.

$\begin{array}{llc} & F 52 & \text { Foln } 3.5 \\ A & 6,210^{3} & 0,710^{3} \\ B & 5,710^{3} & 7,610^{3} \\ C & 0,610^{3} & 8,010^{3}\end{array}$

Experimental and theoretical interactive coefficients and their $5 \%$ confidence intervals.

$\begin{array}{llc} & F 5 & \text { Foln } 3.5 \\ A & 6.210^{3} & 0.710^{3} \\ B & 5.710^{3} & 7.610^{3} \\ C & 0.610^{3} & 8.010^{3}\end{array}$

Bien que de nombreux travaux mathématiques aient été consacrés aux interactions entre 2 populations, les approches expérimentales confrontant données biologiques et modèle sont rares. Il est vraisemblable que l'intérêt du modèle qui vient d'être décrit dépasse le cadre de la compétition entre Fusarium. Sur le même principe d'analyse des dynamiques de populations isolées permettant d'estimer les paramètres de croissance suivies d'expérimentations de confrontation entre 2 populations, nous abordons les études d'interactions entre différentes populations microbiennes du sol, en particulier les relations de compétition entre Fusarium et bactéries du sol. 


\section{RÉFÉRENCES BIBLIOGRAPHIQUES}

Alabouvette C., 1986. Fusarium-wilt suppressive soils from the Châteaurenard region : review of a 10-year study. Agronomie, 6 (3), 273-284.

Amarger N. S., Lobreau J. P., 1982. Quantitative study of nodulation competitiveness in Rhizobium strains. Appl. environ. Microbiol., 44, 583.

Baranger J., 1977. Introduction à l'analyse numérique. Hermann Paris, $125 \mathrm{p}$

Corman A., Couteaudier Y., Zegerman M., Alabouvette C., 1986. Réceptivité des sols aux fusarioses vasculaires : méthode statistique d'analyse des résultats. Agronomie, 6 (8), 751-757.

Corman A., Pavé A., 1983. On parameter estimation of Monod's bacterial growth model from batch culture data. J. gen. appl. Microbiol., 29, 91-101.

Couteaudier Y., Letard M., Alabouvette C., Louvet J., 1985. Lutte biologique contre la fusariose vasculaire de la tomate. Résultats en serre de production. Agronomie, 5 (2), 151-156.

Couteaudier Y., Zegerman M., Alabouvette C., 1986. Dynamique des populations de Fusarium dans les sols : intérêt de cette étude dans le cadre de la lutte biologique. 225-231. In Actes Col. Natl. Biol. Populations. Septembre 86, Lyon I, 699 p.
Finney D. J., 1978. Statistical Method in Biological Assay. Charles Griffin and Co ed., Londres, $3^{\mathrm{e}}$ ed., $463 \mathrm{p}$.

Lotka A. J., 1932. The growth of mixed population : two species competing for a common food supply. J. Washington Acad. Sci., 22, 461-469.

Pavé A., 1980. Contribution à la théorie et à la pratique des modèles mathématiques pour l'analyse dynamique des systèmes biologiques. Etude de quelques cas typiques en biologie cellulaire et moléculaire. Thèse Dr ès-Sciences, Univ. Claude Bernard, Lyon 1.

Pavé A., Rechenmann F., 1986. Computer-aided modelling in biology : an artificial intelligence approach. Simulation Series, 18 (1), $52-66$.

Son M., 1985. Etude de la croissance de Fusarium en culture pure et en confrontation: analyse des données expérimentales et modélisation. D.E.A. Univ. Claude Bernard, Lyon I.

Tello-Marquina J. C., Alabouvette C., 1984. Observations sur la persistance dans les sols des microconidies de Fusarium oxysporum. Agronomie, 4 (9), 885-890.

Volterra L., 1931. Leçons sur la théorie mathématique de la lutte pour la vie. Ed. Gauthier-Villars, Paris, 210. 